\title{
An anatomical study of the spinous process of the seventh cervical vertebrae based on the three-dimensional computed tomography reconstruction
}

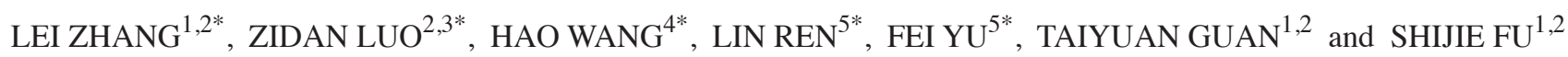 \\ ${ }^{1}$ Department of Orthopedics; ${ }^{2}$ Academician Workstation in Luzhou, Affiliated Traditional Chinese Medicine Hospital of \\ Southwest Medical University; ${ }^{3}$ Department of Clinical Medicine, School of Clinical Medicine, Luzhou, Sichuan 646600; \\ ${ }^{4}$ The Key Laboratory of Molecular Biology, School of Traditional Chinese Medicine, Southern Medical University, \\ Guangzhou, Guangdong 510515; ${ }^{5}$ Department of Radiology, Affiliated Traditional Chinese Medicine \\ Hospital of Southwest Medical University, Luzhou, Sichuan 646600, P.R. China
}

Received November 4, 2017; Accepted January 26, 2018

DOI: $10.3892 /$ etm.2018.6245

\begin{abstract}
Palpation of the seventh cervical vertebra (C7) is important for the diagnosis and treatment of neck and chest conditions. However, the spinous process of C7 (C7-SP) displays an anatomical deviation among individuals. The present study aimed to clarify anatomic characteristics of C7-SP by using a three-dimensional (3D) computed tomographic (CT) reconstruction technique. A total of 245 subjects meeting the selection criteria were examined. After CT scanning, the images were reconstructed in 3D. All subjects were grouped according to their deviation of C7-SP: Deviating to the right (DR group), deviating to the left (DL group) and no deviation (ND group). Three distances and three angles were recorded on C7-SP. The vertical distances between the borders of the left and right transverse processes and the tip of the SP, were termed DLTS and DRTS, respectively. The length of the SP was also determined. The angle of the SP deviation was referred to as $\angle \alpha$, the angle between the SP axis
\end{abstract}

Correspondence to: Professor Shijie Fu, Academician Workstation in Luzhou, Affiliated Traditional Chinese Medicine Hospital of Southwest Medical University, 182 Chun Hui Road, Luzhou, Sichuan 646600, P.R. China

E-mail: fushijieggj@126.com

*Contributed equally

Abbreviations: C7, seventh cervical vertebra; SP, spinous process; 3D CT, three dimensional computed tomography; DL, deviating to left; DR, deviating to right; ND, no deviation; DLTS, distance between the left transverse process and spinous process; DRTS, distance between the right transverse process and spinous process; LSP, length of SP; PACS, picture archiving communication system

Key words: seventh cervical vertebra, spinous process, three-dimensional computed tomography, reconstruction, anatomic deviation and the line crossing the tips of the transverse processes was referred to as $\angle \beta$ and the angle between the vertebral body axis and the SP axis was referred to as $\angle \gamma$. Among the three groups, differences in $\angle \alpha$ and $\angle \beta$ were statistically significant $(\mathrm{P}<0.05)$. Furthermore, the DLTS was significantly different between the DL and ND groups $(\mathrm{P}<0.05)$. In addition, a significant difference in the DRTS was identified between the DR and ND groups $(\mathrm{P}<0.05)$. 3D CT reconstruction was reliable for studying anatomic characteristics of C7-SP. Based on this, patients may be preliminarily grouped according to the deviation of their C7-SP and the measurement of the C7-SP may guide clinical diagnoses and treatments.

\section{Introduction}

Upon lowering the head, the spinous process (SP) of the seventh cervical vertebra (C7) of humans bulges to the skin and forms a significant eminence on the back of the neck. The C7 is usually identified by a long, club-shaped and unbranched SP (1-3), referred to as vertebra prominens (4). The C7 represents the bottom of the cervical spine and connects with the top of the thoracic spine, T1, to form the cervicothoracic junction, also referred to as C7-T1. Thus, the C7 marks the level of transition from the dynamic cervical segment to the relatively inflexible thoracic segment of the vertebral column (1). Furthermore, it is the point where cervical lordosis reverses into thoracic kyphosis, making C7 anatomically unique (5). Of all cervical injuries, $9 \%$ occur at the C7 segment, and the morphology of $\mathrm{C}$, which displays inter-regional, inter-ethnic and individual variations, may affect surgical approaches (6). Thus, the morphology of the $\mathrm{C} 7$ is a clinically significant area of interest in clinical practice (1).

In addition, C7-SP is of vital importance for clinical examinations, diagnostic and therapeutic interventions and various types of surgery involving the neck. For instance, C7-SP is used as a point of reference for determining the level to insert epidural catheters by anesthesiologists, and a C7-T1 level of entry is recommended for cervical interlaminar epidural steroid injection (7). In addition, owing to the 
complex attachment of nuchal muscles to the spinous process, the SP should be preserved to reduce the incidence of postoperative axial pain (8). For the most part, decisions regarding surgical treatment made by the physician are affected by the anatomic characteristics of C7-SP. For instance, when performing posterior cervical surgeries, surgeons often rely on the morphology of C7-SP if the operation only involves the lower cervical area. However, if the patient has a bifid C7-SP, this may mislead the surgeons, which may result in undesired outcomes (2). In addition, due to the inter-individual deviation of C7-SP, the identification of suitable approaches for inserting the screws when performing a posterior fixation of the neck is challenging. Certain studies stressed that a new pedicle screw should be inserted based on the three-dimensional (3D) reconstruction as well as individualization (9). Although numerous studies have assessed the structures of C7-SP, most of them focused on the dimensions and angulations of the pedicle, bifid condition and the accurate ways to locate C7 (10-13). However, the detailed anatomic characteristics of $\mathrm{C} 7-\mathrm{SP}$ have remained to be elucidated.

Only a few reports have assessed the inter-individual variation of C7-SP. In the present study, the anatomic characteristics of the C7-SP were preliminarily measured based on 3D computed tomography (CT) reconstruction, an imaging tool that has been validated in a variety of applications for the practice of spine surgery (14). The objective of the present study was to explore the anatomical features of C7-SP, which may facilitate the diagnosis and treatment of conditions involving the cervical spine.

\section{Patients and methods}

Subjects. A total of 245 subjects were enrolled from January 2016 to August 2017 at the Affiliated Traditional Chinese Medicine Hospital of Southwest Medical University (Luzhou, China). Prior to the start of the study, approval was obtained from the Ethical Inspection Committee of the Affiliated Traditional Chinese Medicine Hospital of Southwest Medical University, which waived the requirement for informed consent due to the retrospective nature. The CT data of the C7 were collected at the Radiology Department of the Affiliated Traditional Chinese Medicine Hospital of Southwest Medical University. All patients were Han Chinese and $>18$ years of age at the time of the CT scan. The majority of the patients had been given a health examination, and the rest were given a special examination the spine, as they had fallen prior to recruitment or suffered accidental impacts, which may detrimentally affect the spine. Individuals with congenital spinal malformation, spinal pathology (including spondylolisthesis, retrolisthesis or disc space collapse), spinal variation and those who had undergone spinal surgeries were excluded.

$3 D$ reconstruction $C T$. A spiral CT scanner (Somatom Emotion; Siemens AG, Munich, Germany) was used with the following scan conditions: Voltage, $130 \mathrm{kV}$; current, $180 \mathrm{~mA}$; thickness, $0.75 \mathrm{~mm}$; and matrix size, $512 \times 512$. In addition, the CARE Dose 4D technique was used in the examination. All patients were kept in the supine position during scanning. The 3D images were stored on the Picture Archiving Communication System (PACS version 4.0; DJ HealthUnion
Table I. Number of males and females, and their age in the three groups.

\begin{tabular}{lcll}
\hline Group & Male & Female & Age (years) \\
\hline DL & $43(17.55)$ & $51(20.82)$ & $47.23 \pm 12.78$ \\
DR & $64(26.12)$ & $69(28.16)$ & $47.95 \pm 14.27$ \\
ND & $5(2.04 \%)$ & $13(5.31)$ & $47.95 \pm 14.27$ \\
\hline
\end{tabular}

Values are expressed as the mean \pm standard deviation or as $\mathrm{n}(\%)$. There was no statistically significant difference in the age and the male-to-female ratio among the three groups ( $\mathrm{P}>0.05)$. Groups: DL, C7-SP deviating to left; DR, C7-SP deviating to right; ND, no deviation in C7-SP. C7-SP, spinous process of the seventh cervical vertebra.

Systems Corporation, Shanghai, China). This was a system for recording and storing radiographic images, permitting storage of large numbers of images and allowing access from any networked station. In addition to these storage facilities, PACS also incorporated a sensitive measuring tool. After 3D reconstruction, the $\mathrm{C} 7$ was individually analyzed. Typical C7 images were acquired by adjusting the position and size, increasing the contrast and making other image adjustments on the CT workstation prior to measuring.

Measurement of C7-SP parameters. All measurements were performed by three radiologists who had performed in CT-associated work for $>5$ years at the Radiology Department of the Affiliated Traditional Chinese Medicine Hospital of Southwest Medical University. The lateral, superior and posterior aspects of $\mathrm{C} 7$ images were saved separately in the PACS. Prior to measurement, the $\mathrm{C} 7$ was placed in different planes for each parameter. The minimal distance was $0.01 \mathrm{~cm}$ and the minimal angle was $1^{\circ}$. The distance between the borders of the left or right transverse process and the tip of SP in the superior aspect (DLTS or DRTS), and the distance between the tip of C7-SP and the medial point of the rear of spinal canal in lateral aspect, termed the length of SP (LSP), were determined. The angle of the SP deviation $(\angle \alpha)$ was measured as the angle between the long axis of SP and the median line of the vertebra in the superior aspect. $\angle \beta$ was defined as the angle between the long axis of the SP and the line that crossed the tips of bilateral transverse processes. $\angle \gamma$ was measured as the angle between the vertical axis and the long axis of the SP in the lateral aspect. All of the abovementioned measurements are indicated in Fig. 1. All subjects of the present study were grouped according to the deviational direction of C7-SP: Deviating to the right (DR group), deviating to the left (DL group) and no deviation (ND group) (Fig. 2).

Statistical analysis. A statistical analysis was performed using SPSS, version 20.0 (IBM Corp., Armonk, NY, USA). The amounts of males and females in each group were expressed as frequencies and percentages and were analyzed by the $\chi^{2}$ test. The age and parameters of C7 were expressed as the mean \pm standard deviation. In order to determine the normal distribution, the Kolmogorov-Smirnov and Shapiro-Wilk tests 

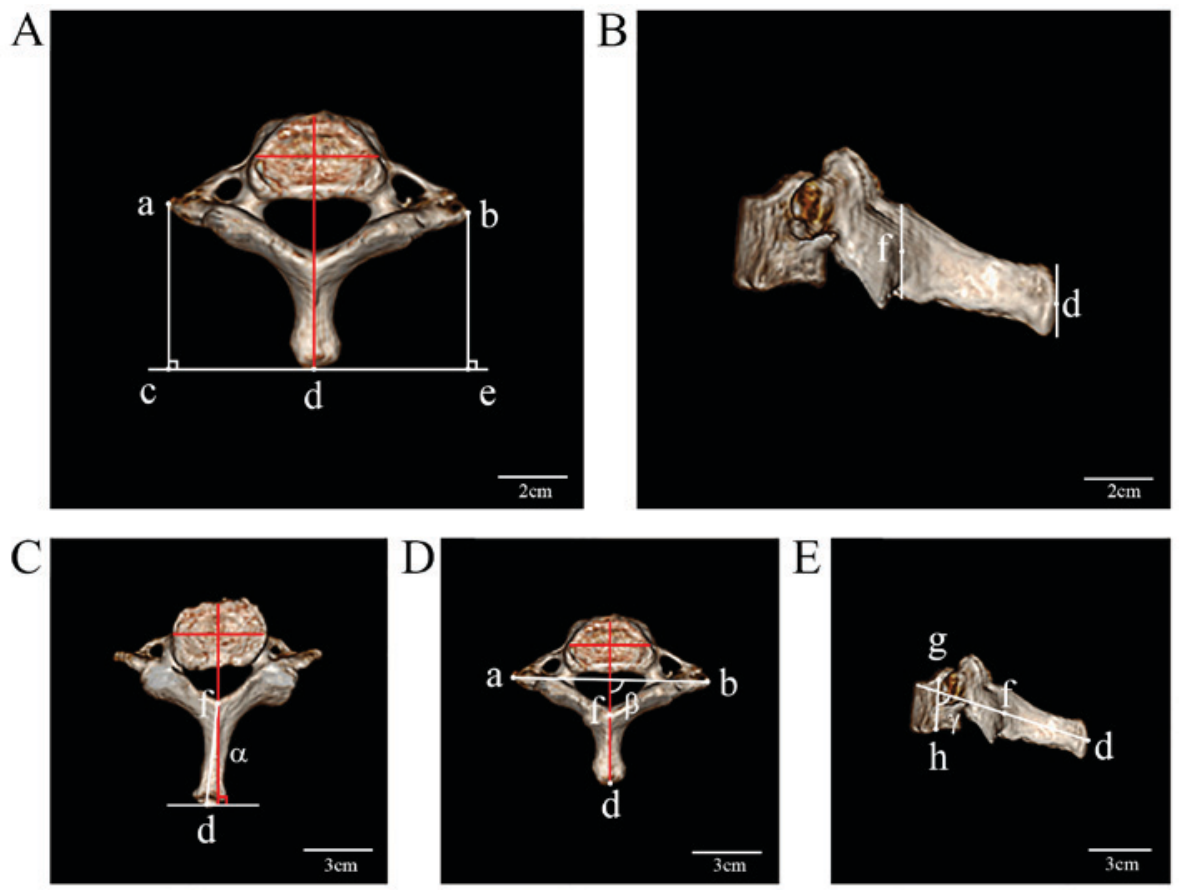

Figure 1. Measurement of the three parameters and three angles of the spinous process of the seventh cervical vertebra in the superior and lateral views. Representative images in the superior view of the (A and D) ND group and (C) DL group, and (B and E) in the lateral view of the DR group. DL, C7-SP deviating to left; DR, C7-SP deviating to right; ND, no deviation in C7-SP; a, the border of the left transverse process; b, the border of the right transverse process; $d$, the tip of the SP; a-c, the vertical distance between the left transverse process to the tip of the SP; b-e, the vertical distance between the border of the right transverse process and the tip of the SP; f, the medial point of the rear of spinal canal; d-f, the distance between the tip of the C7-SP and the medial point of the rear of the spinal canal in the lateral aspect; $g$-h, the axis of the vertebral body. $\angle \alpha$, the angle between the long axis of the SP and the median line of the vertebra in the superior aspect; $\angle \beta$, the angle between the long axis of the SP and the line which crossed the tips of the bilateral transverse processes in the superior aspect; $\angle \gamma$, the angle between the vertical body axis and the SP axis in the lateral aspect.
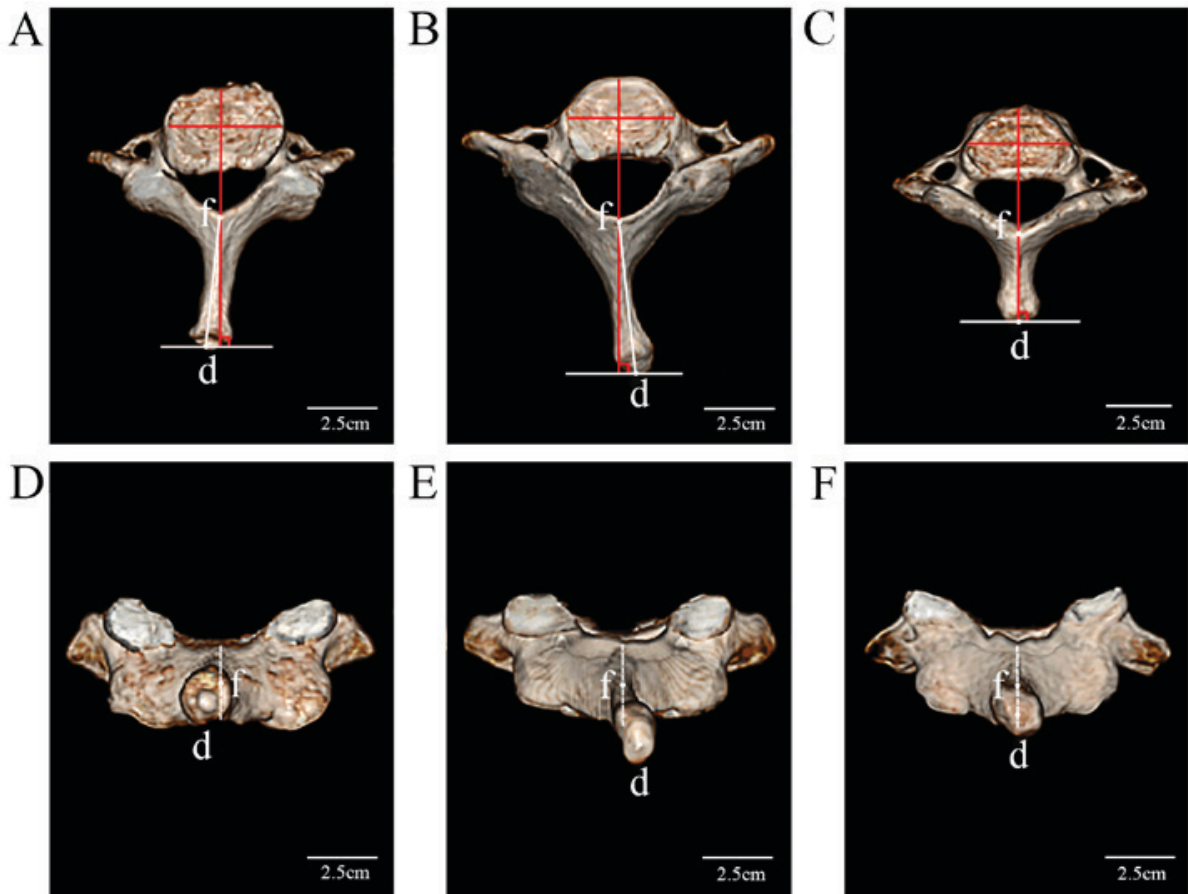

Figure 2. Different deviations of the C7-SP in the three groups in superior and posterior view, respectively. Representative images in the superior view of the (A) DL group, (B) DR group and (C) ND group, and in the posterior view of the (D) DL group, (E) DR group and (F) ND group. DL, C7-SP deviating to left; DR, C7-SP deviating to right; ND, no deviation in C7-SP; d, tip of the SP; f, medial point of the rear of the spinal canal; d-f, distance between the tip of the C7-SP and the medial point of the rear of the spinal canal in the lateral aspect; C7-SP, spinous process of the seventh cervical vertebra.

were performed. One-way analysis of variance was used to compare the age and parameters of $\mathrm{C} 7$ among the three groups, and the Student-Newman-Keuls test was the post hoc test. $\mathrm{P}<0.05$ was considered to indicate a statistically significant difference. 
Table II. Measurements in the three groups.

\begin{tabular}{|c|c|c|c|c|c|c|}
\hline Group & DLTS $(\mathrm{cm})$ & DRTS $(\mathrm{cm})$ & $\mathrm{LSP}(\mathrm{cm})$ & $\angle \alpha\left(^{\circ}\right)$ & $\angle \beta\left(^{\circ}\right)$ & $\angle \gamma\left({ }^{\circ}\right)$ \\
\hline DL & $4.69 \pm 0.53^{\mathrm{b}}$ & $4.59 \pm 0.51$ & $3.03 \pm 0.37$ & $4.30 \pm 2.96^{\mathrm{a}, \mathrm{b}}$ & $93.09 \pm 3.39^{\mathrm{a}, \mathrm{b}}$ & $70.95 \pm 7.96$ \\
\hline DR & $4.57 \pm 0.50$ & $4.72 \pm 0.47^{b}$ & $3.08 \pm 0.36$ & $-5.17 \pm 3.35^{\mathrm{b}}$ & $86.30 \pm 3.70^{\mathrm{b}}$ & $69.33 \pm 10.04$ \\
\hline ND & $4.41 \pm 0.54$ & $4.44 \pm 0.45$ & $2.98 \pm 0.28$ & $0.00 \pm 0.00$ & $89.72 \pm 2.02$ & $70.64 \pm 8.13$ \\
\hline
\end{tabular}

${ }^{\mathrm{a} P}<0.05$ vs. DR; ${ }^{\mathrm{P}}<0.05$ vs. ND. Values are expressed as the mean \pm standard deviation. Groups: DL, C7-SP deviating to left; DR, C7-SP deviating to right; ND, no deviation in C7-SP. DLTS/DRTS, vertical distances between the borders of the left/right transverse processes and the tip of SP, respectively, in the superior aspect; LSP, the distance between the tip of the C7-SP and the rear of the spinal canal in lateral aspect; $\angle \alpha$, angle of SP deviation, measured as the angle between the long axis of the SP and the median line of the vertebra in the superior aspect; $\angle \beta$, angle between the long axis of the SP and the line which crossed the tips of the bilateral transverse processes in the superior aspect; $\angle \gamma$, angle between the vertical body axis and the SP axis in the lateral aspect; C7-SP, spinous process of the seventh cervical vertebra.

\section{Results}

A total of 245 subjects were enrolled in the present study, and the number of patients in the DL group was 94, which comprised 43 males $(17.55 \%)$ and 51 females $(20.82 \%)$, and the average age was $47.23 \pm 12.78$ years (range, $21-80$ years). The DR group consisted of 133 patients with an average age of 47.95 \pm 14.27 years (range, 19-89 years), and included 64 males (26.12\%) and 69 females (28.16\%). The ND group included 18 subjects, comprising 5 males (2.04\%) and 13 females $(5.31 \%)$ with an average age of $47.95 \pm 14.27$ years (range, 25-86 years). No statistically significant difference in the age and the male-to-female ratio among the three groups was determined (Table I).

A statistically significant difference in the DLTS between the DL and ND groups was observed (4.69 \pm 0.53 vs. $4.41 \pm 0.54 \mathrm{~cm}$; $\mathrm{P}<0.05)$. In addition, the DRTS in the DR group was significantly different from that in the ND group (4.72 \pm 0.47 vs. $4.44 \pm 0.45 \mathrm{~cm} ; \mathrm{P}<0.05)$. Compared with that in the ND group, the $\angle \alpha$ in the DL and DR groups was significantly different ( 0 vs. $4.30 \pm 2.96$ and $-5.17 \pm 3.35^{\circ} ; \mathrm{P}<0.05$ ); furthermore, the $\angle \alpha$ in the DL group was significantly different from that in the DR group $(\mathrm{P}<0.05)$. More importantly, $\angle \beta$ was significantly different among the DL, DR and ND groups (93.09 \pm 3.39 , $86.30 \pm 3.70$ and $89.72 \pm 2.02^{\circ}$, respectively; $\left.\mathrm{P}<0.05\right)$. The differences in the LSP and $\angle \gamma$ among the three groups were not significant $(\mathrm{P}>0.05$; Table II).

\section{Discussion}

The accuracy in identifying C7 using manual palpation by clinicians is limited due to the inter-individual variation in the morphology of C7-SP (15-19). While several studies have assessed the anatomic structures of C7-SP, most of them focus on the mechanisms of diseases affecting it $(8,20,21)$. The SP extends from the posterior of the vertebral arch to provide connection points for the muscles that extend from the neck, including the trapezius and spinalis muscles. The end of the nuchal ligament, which supports the muscles of the neck and connects the occipital bone of the skull to the $\mathrm{C} 7$ vertebra, attaches at the tip of the spinous process. According to various studies, the preservation of C7-SP has a crucial role in preventing axial symptoms (22-25). This is a form of pain around the neck and shoulders and often remains a major concern for several years postoperatively, even in patients with excellent neurologic recovery $(26,27)$. In addition, protection of the C7-SP and anatomical structures around the cervical spine, including the muscles attached to the spinous process, as well as the supraspinous and interspinous ligaments, provides better results regarding the range of movement and cervical axial pain (28). In addition, it was reported that adequate knowledge of $\mathrm{C} 7$ morphology is necessary for the spinal surgeon in order to avoid damage to the vertebral arteries, spinal cord or nerve roots during fixation interventions involving the posterior cervical spine (29). The cervicothoracic junction is a challenging anatomical transition in spine surgery. Compared with all other cervical vertebrae, $\mathrm{C} 7$ has relatively broader laminae, larger pedicles, smaller lateral masses and a long non-bifid spinous process. These characteristics allow for a variety of surgical methods to be performed to apply posterior rigid instrumentation in the form of different types of screw, including lateral mass, pedicle, transfacet and intralaminar screws (1).

In the present study, all subjects were grouped according to the angle of SP deviation. Compared with that in the ND group, the $\angle \alpha$ of the DL and DR groups was significantly different. It was indicated that a deviation of the C7-SP existed, and that it was possible to preliminarily group all $\mathrm{C} 7 \mathrm{~s}$ according to their $\angle \alpha$. Furthermore, as the age and male-to-female ratio did not significantly differ between the groups, the grouping was not affected by the age and the male-to-female ratio.

After the grouping, no significant differences were noted in $\angle \gamma$ and LSP among the three groups $(\mathrm{P}>0.05)$. Numerous other studies had been performed to measure the LSP. Bazaldua et al (30), studied the morphometry of the cervical vertebrae C3-7 in a population from Northeastern Mexico and measured the distance from the superior border to the tip of the SP in the sagittal plane to determine a mean value of $29.12 \pm 5.86 \mathrm{~mm}$, which was in accordance with the results of the present study. However other studies obtained measures of 22.19 \pm 2.02 and $22.78 \pm 2.03 \mathrm{~mm}(31,32)$, which was smaller than the result of the present study. These discrepancies may be the result of the various measuring criteria, inter-regional or -ethnic differences and statistical errors.

Furthermore, in the present study, the difference in the DLTS between the DL and ND groups was statistically significant $(4.69 \pm 0.53$ vs. $4.41 \pm 0.54 \mathrm{~cm} ; \mathrm{P}<0.05)$. A statistically significant difference in the DRTS between the DR and 
ND groups was also observed $(4.72 \pm 0.47$ vs. $4.44 \pm 0.45 \mathrm{~cm}$; $\mathrm{P}<0.05)$. This indicated that the orientation that SP deviated to had a longer distance between the transverse process and SP. This may be due to the rotation of the vertebral body. Stricter measuring methods are required to be formulated to reduce these errors. More importantly, statistically significant differences were also observed in $\angle \beta$ among the three groups $(\mathrm{P}<0.05)$. This parameter reflects the angle between the transverse process and SP, and it is of vital importance for surgeries involving the exposure of the posterolateral part of C7. In addition, it was reported that the most effective type of posterior fixation at the cervicothoracic junction was a pedicle screw fixation due to the specific characteristics of the C7 (7,33-36). Kajino et al (37), studied the surgical anatomy for pedicle screw placement in the cervical spine. They confirmed that the ideal posterior entrance for screw insertion was $2.5 \mathrm{~mm}$ medial to the lateral margin of the lateral masses, the correct insertion angle was $\sim 45^{\circ}$ and the critical value of depth of insertion was 13-14 mm. Lee et al (38), studied the anatomic feasibility of posterior cervical pedicle screw placement in children with CT analysis, arguing that the possibility of subaxial lateral mass screw fixation must be investigated with a more tailored method. In this study, before the measurement, all the objectives accepted CT scan. The value of CT in detecting spinal fractures is well known. Unlike conventional X-ray analysis, CT scanning provides detailed images of numerous types of tissue as well as the bones and blood vessels. Thus, CT has been used in clinical fields including diagnosis of diseases and anatomical analysis (39-41). According to the Food and Drug Administration, CT scanning is a rapid procedure and offers accurate evaluation of bone and most soft tissues. Using the latest equipment, the spine may be displayed in multiple planes and 3D images may be reconstructed. In the present study, the scan images were reconstructed in $3 \mathrm{D}$ models. Compared with conventional 2D images, 3D images may provide more advancement in the clinic. In addition, these CT images were taken from helical CTs, which provided adequate data for creating 3D images.

The present study had certain limitations. First, the parameters of the C7-SP were measured on the PACS, in which the tips or medial parts of the structures were determined by three radiologists. A more advanced measuring tool is required to provide accurate results. In addition, only the C7-SP of Chinese patients from the Affiliated Traditional Chinese Medicine Hospital of Southwest Medical University were assessed, and patients aged $<18$ years were excluded; further studies may be performed at other hospitals for other populations/ethnicities and underage subjects. Furthermore, no cadaveric study of the C7-SP was performed in the present study, which may be the aim of a future study.

In conclusion, based on the $3 \mathrm{D} \mathrm{CT}$, several parameters of the C7-SP were precisely measured. It was identified that despite of the different age and gender of the patients, it was possible to preliminarily group all of them according to the deviation of C7-SP. These results may support future diagnoses and treatments, and reduce the incidence of misdiagnosis. In addition, the present study may provide a basis for further studies on C7. Apart from the deviation of C7-SP, it remains to be determined whether there is an association with any other parameters. Therefore, more study is required for assessing the association between C7-SP, the bony structures and tissues surrounding it.

\section{Acknowledgements}

Not applicable.

\section{Funding}

This work was supported by Academician Workstation Construction Project of Luzhou (Luzhou, China; grant no. 20180101).

\section{Availability of data and materials}

The analysed data sets generated during the study are available from the corresponding author on reasonable request.

\section{Authors' contributions}

The study was designed by LZ. Patients were recruited by ZL, HW, LR, FY, TG and SF. The imaging measurements were made by ZL, LR and FY. The statistical analysis was conducted by LZ and HW. LZ, ZL, TG and SF wrote the manuscript.

\section{Ethical approval and consent to participate}

All of the procedures were approved by the Ethical Committee of the Affiliated Traditional Chinese Medicine Hospital of Southwest Medical University (Luzhou, China; no. SWMCTCM2017-0810) and registered as a clinical trial (ChiCTR-BOC-17012270), and were performed in accordance with the 1964 Helsinki declaration and its later amendments or comparable ethical standards. Informed consent was obtained from all individual participants included in the study.

\section{Consent for publication}

Not applicable.

\section{Competing interests}

The authors declare that they have no competing interests.

\section{References}

1. Bayoumi AB, Efe IE, Berk S, Kasper EM, Toktas ZO and Konya D: Posterior rigid instrumentation of C7: Surgical considerations and biomechanics at the cervicothoracic junction. A Review of the literature. World Neurosurg 111: 216-226, 2018.

2. Cho W, Maeda T, Park Y, Buchowski JM, Nabb CE and Riew D: The incidence of bifid C7 spinous processes. Global Spine J 2: 99-104, 2012.

3. Greiner TM: Shape analysis of the cervical spinous process. Clin Anat 30: 894-900, 2017.

4. Asvat R: The configuration of cervical spinous processes in black and white South African skeletal samples. J Forensic Sci 57: 176-181, 2012.

5. Hong JT, Yi JS, Kim JT, Ji C, Ryu KS and Park CK: Clinical and radiologic outcome of laminar screw at $\mathrm{C} 2$ and $\mathrm{C} 7$ for posterior instrumentation-review of 25 cases and comparison of $\mathrm{C} 2$ and C7 intralaminar screw fixation. World Neurosurg 73: 112-118, 2010. 
6. Tse MS, Chan $\mathrm{CH}$, Wong KK and Wong WC: Quantitative anatomy of $\mathrm{C} 7$ vetebra in southern chinese for insertion of lateral mass screwsand pedicle screw. Asian Spine J 10: 705-710, 2016

7. Kothe R, Ruther W, Schneider E and Linkeet B: Biomechanical analysis of transpedicular screw fixation in the subaxial cervical spine. Spine (Phila Pa 1976) 29: 1869-1875, 2004.

8. Ono A, Tonosaki Y, Yokoyama T, Aburakawa S, Takeuchi K, Numasawa T, Wada K, Kachi T and Toh S: Surgical anatomy of the nuchal muscles in the posterior cervicothoracic junction: Significance of the preservation of the $\mathrm{C} 7$ spinous process in cervical laminoplasty. Spine (Phila Pa 1976) 33: E349-E354, 2008.

9. Liao W, Guo L, Bao H and Wanget L: Morphometric analysis of the seventh cervical vertebra for pedicle screw insertion. Indian J Orthop 49: 272-277, 2015.

10. Barrey C, Cotton F, Jund J, Mertens P and Perrin G: Transpedicular screwing of the seventh cervical vertebra: Anatomical considerations and surgical technique. Surg Radiol Anat 25: 354-360, 2003.

11. Tomasino A, Parikh K, Koller H, Zink W, Tsiouris AJ Steinberger J and Härtl R: The vertebral artery and the cervical pedicle: Morphometric analysis of a critical neighborhood. J Neurosurg Spine 13: 52-60, 2010.

12. Bruneau M, Cornelius JF, Marneffe V, Triffaux M and George B: Anatomical variations of the V2 segment of the vertebral artery. Neurosurgery 59: ONS20-ONS24, 2006.

13. Jovanoviic MS: A comparative study of the foramen transversarium of the sixth and seventh cervical vertebrae. Surg Radiol Anart 12: 167-172, 1990.

14. Schell A, Rhee JM, Holbrook J, Lenehan E and Park KY: Assessing foraminal stenosis in the cervical spine: A comparison of three-dimensional computed tomographic surface reconstruction to two-dimensional modalities. Global Spine J 7: 266-271, 2017.

15. Shin S, Yoon DM and Yoon KB: Identification of the correct cervical level by palpation of spinous processes. Anesth Analg 112: 1232-1235, 2011.

16. Ingram LA, Snodgrass SJ and Rivett DA: Comparison of cervical spine stiffness in individuals with chronic nonspecific neck pain and asymptomatic individuals. J Orthop Sports Phys Ther 45: $162-169,2015$.

17. Robinson R, Robinson HS, Bjorke G and Kvaleet A: Reliability and validity of a palpation technique for identifying the spinous processes of C7 and L5. Man Ther 14: 409-414, 2009.

18. Hogan Q: Anatomy of the neuraxis. In: Cousins MJ, Carr DB Horlocker TT, Bridenbaugh TH, (eds). Cousins and Bridenbaugh's Neural Blockade in Clinical Anesthesia and Pain Medicine. 4th edition. Philadelphia, Lippincott Williams \& Wilkins, pp181-212, 2009

19. Cooperstein R and Haneline MT: Spinous process palpation using the scapular tip as a landmark vs. a radiographic criterion standard. J Chiropr Med 6: 87-93, 2007.

20. Zenmyo M, Komiya S, Hamada T and Inouee A: A solitary bone cyst in the spinous process of the cervical spine: A case report. Spine (Phila Pa 1976) 25: 641-642, 2000.

21. Zhang P, Shen Y, Zhang YZ, Ding WY, Xu JX and Cao JM: Preserving the $\mathrm{C} 7$ spinous process in laminectomy combined with lateral mass screw to prevent axial symptom. J Orthop Sci 16: 492-497, 2011

22. Takeuchi $\mathrm{T}$ and Shono Y: Importance of preserving the $\mathrm{C} 7$ spinous process and attached nuchal ligament in French-door laminoplasty to reduce postoperative axial symptoms. Eur Spine J 16: 1417-1422, 2007.

23. Umeda M, Sasai K, Kushida T, Wakabayashi E, Maruyama T, Ikeura A and Iida $\mathrm{H}$ : A less-invasive cervical laminoplasty for spondylotic myelopathy that preserves the semispinalis cervicis muscles and nuchal ligament. J Neurosurg Spine 18: 545-552, 2013.

24. Mori E, Ueta T, Maeda T, Yugué I, Kawano O and Shiba K: Effect of preservation of the C-6 spinous process and its paraspinal muscular attachment on the prevention of postoperative axial neck pain in C3-6 laminoplasty. J Neurosurg Spine 22: 221-229, 2015
25. Kato M, Nakamura H, Konishi S, Dohzono S, Toyoda H, Fukushima W, Kondo K and Matsuda H: Effect of preserving paraspinal muscles on postoperative axial pain in the selective cervical laminoplasty. Spine (Phila Pa 1976) 33: E455-E459, 2008

26. Zhang P, Shen Y, Zhang YZ, Ding WY, Xu JX and Cao JM Preserving the $\mathrm{C} 7$ spinous process in laminectomy combined with lateral mass screw to preventaxial symptom. J Orthop Sci 16: 492-497, 2011

27. Hosono N, Sakaura H, Mukai Y, Fujii R and Yoshikawa H: C3-6 laminoplasty takes over C3-7 laminoplasty with significantly lower incidence of axial neck pain. Eur Spine J 15: 1375-1379, 2006.

28. Secer HI, Harman F, Aytar MH and Kahraman S: Open-door laminoplasty with preservation of muscle attachments of $\mathrm{C} 2$ and C7 for cervical spondylotic myelopathy, retrospective study. Turk Neurosurg 28: 257-262, 2018.

29. Karaikovic EE, Kunakornsawat S, Daubs MD, Madsen TW and Gaines RW Jr: Surgical anatomy of the cervical pedicles: Landmarks for posterior cervical pedicle entrance localization. J Spine Disord 13: 63-72, 2000.

30. Bazaldúa CJJ, González LA, Gómez SA, Villarreal SE, Velázquez GSE, Sánchez UA, Elizondo-Omaña RE and Guznán LS: Morphometric study of cervical vertebrae C3-C7 in a population from Northeastern Mexico. Int J Morphol 29: 325-330, 2011

31. Parashar R, Saxena D, Chauhan S, Arora R and Abhijeet J: A morphometric study of pedicle, lamina and spinous process of C3-C7 vertebrae in Rajasthan population. Int J Res Med 3: 140-145, 2014.

32. Prabavathy G, Philip XC, Arthi G and Sadeesh T: Morphometric study of cervical vertebrae C3-C7 in South Indian population-A clinicoanatomical approach. Italian J Anat Embryol 122: 49-57, 2017.

33. Rhee JM, Kraiwattanapong C and Hutton WC: A comparison of pedicle and lateral mass screw construct stiffnesses at the cervicothoracic junction: A biomechanical study. Spine (Phila Pa 1976) 30: E636-E640, 2005.

34. Ramos-Peek J: Alarm criteria for cervical, thoracic and lumbar pedicle crews. Clini Neurophysiol 127: e304-e305, 2016.

35. Wu C, Chen C, Wu W, Zhao W, Sun P, Fan J, Bi Z, Zhang J and Ouyang J: Biomechanical analysis of differential pull-out strengths of bone screws using cervical anterior transpedicular technique in normal and osteoporotic cervical cadaveric spines. Spine (Phila Pa 1976) 40: E1-E8, 2015.

36. Fan D, Song R, Zhang M, Bai R, Li Y, Zhang Z, Wu H, Wang Y, Zhao L, Gao W, et al: Guideline for $\mathrm{C} 1$ lateral mass and $\mathrm{C} 2$ pedicle screw choices in children younger than 6 years. Spine (Phila Pa 1976) 42: E949-E955, 2017.

37. Kajino T, Taneichi H, Suda K and Kaneda K: Surgical Anatomy for Pedicle Screw Placement in the Cervical Spine. J Japan Spine Res Soc 15: 141-142, 2004.

38. Lee H, Hong JT, Kim IS, Kim MS, Sung JH and Lee SW: Anatomic feasibility of posterior cervical pedicle screw placement in children: Computerized tomographic analysis of children under 10 years old. J Korean Neurosurg Soc 56: 475-481, 2014.

39. Melamed KH, Abtin F, Barjaktarevic I and Cooper CB Diagnostic value of quantitative chest CT scan in a case of spontaneous pneumothorax. Chest 152: e109-e114, 2017.

40. Feng ST, Zhu H, Peng Z, Huang L, Dong Z, Xu L, Huang K, Yang X, Lin Z and Li ZP: An individually optimized protocol of contrast medium injection in enhanced CT scan for liver imaging. Contrast Media Mol Imaging 2017: 7350429, 2017.

41. Riaz S, Naz F, Bashir H and Niazi IK: 'Bottle Brush Sign'-spinal meningeal disease on 18F-FDG PET-CT scan. Clin Nucl Med 41: 726-727, 2016.

This work is licensed under a Creative Commons Attribution-NonCommercial-NoDerivatives 4.0 International (CC BY-NC-ND 4.0) License. 